\title{
BMJ Open Safety of furazolidone-containing regimen in Helicobacter pylori infection: a systematic review and meta-analysis
}

\author{
Chao-ran Ji, ${ }^{1,2}$ Jing Liu, ${ }^{1,2}$ Yue-yue Li, ${ }^{1,2}$ Chuan-guo Guo, ${ }^{3}$ Jun-yan Qu, ${ }^{1,2}$ \\ Yan Zhang, ${ }^{1,2}$ Xiuli Zuo (iD ${ }^{1,2}$
}

To cite: Ji C, Liu J, Li Y, et al. Safety of furazolidonecontaining regimen in Helicobacter pylori infection: a systematic review and meta-analysis. BMJ Open 2020;10:e037375. doi:10.1136/ bmjopen-2020-037375

- Prepublication history and additional material for this paper are available online. To view these files, please visit the journal online (http://dx.doi. org/10.1136/bmjopen-2020037375).

Received 30 January 2020 Revised 07 August 2020 Accepted 22 August 2020

Check for updates

(C) Author(s) (or their employer(s)) 2020. Re-use permitted under CC BY-NC. No commercial re-use. See rights and permissions. Published by BMJ.

\section{${ }^{1}$ Department of}

Gastroenterology, Qilu Hospital, Cheeloo College of Medicine, Shandong University, Jinan, Shangdong, China

${ }^{2}$ Laboratory of Translational Gastroenterology, Qilu Hospital, Cheeloo College of Medicine, Shandong University, Jinan, Shandong, China

${ }^{3}$ Department of Medicine, University of Hong Kong, Hong Kong, China

Correspondence to

Dr Xiuli Zuo;

zuoxiuli@sdu.edu.cn

\section{ABSTRACT}

Objectives Furazolidone containing regimen is effectivefor Helicobacter pylori (H. pylori) infection, but its safetyremains controversial. To assess the safety of furazolidone containing regimenin $H$. pylori infection. Design A systematic review and meta-analysis. Data sources PubMed, Embase, Cochrane Library, Web of Science and Scopus databases were systematically searched for eligible randomised controlled trials.

Eligibility criteria Studies comparing furazolidone with non-furazolidone-containing regimen, variable durations or doses of furazolidone were included.

Data extraction and synthesis Two reviewers independently selected studies and extracted data. Primary outcomes were the risk of total adverse events (AEs), serious AEs and severe AEs, expressed as relative risk (RR) with $95 \% \mathrm{Cl}$. Secondary outcomes contained the incidence of individual adverse symptoms, AE-related treatment discontinuation and compliance.

Results Twenty-six articles were identified from 2039 searched records, of which 14 studies $(n=2540)$ compared furazolidone with other antibiotics. The eradication rates of furazolidone-containing regimen were higher than those of other antibiotics in both intention-to-treat (RR 1.06, $95 \% \mathrm{Cl} 1.01$ to 1.12) and per-protocol analysis (RR 1.05, $95 \% \mathrm{Cl} 1.00$ to 1.10). Only two serious AEs were reported in furazolidone group $(2 / 1221,0.16 \%)$. No significant increased risk was observed for the incidence of total AEs (RR 1.04, 95\% Cl 0.89 to 1.21) and severe AEs (RR 1.81, $95 \% \mathrm{Cl} 0.91$ to 3.60 ). Twelve studies ( $n=3139$ ) compared different durations of furazolidone, and four studies $(n=343)$ assessed variable doses. Elevated risk of total AEs and severe AEs were only found in a high daily dose of furazolidone rather than prolonged duration. The incidence of AE-related treatment discontinuation and compliance of patients were all similar, irrespective of dose and duration adjustments.

Conclusion Furazolidone-containing regimen has a similar risk of AEs and compliance as non-furazolidonecontaining regimen. A low daily dose of $200 \mathrm{mg}$ is well-tolerated for 14 day regimen and should be first considered.

PROSPERO registration number CRD42019137247

\section{INTRODUCTION}

Helicobacter pylori infection affects up to $44.3 \%$ of the world's population. ${ }^{1}$ Approximately

\section{Strengths and limitations of this study}

This review screens trials in both initial and rescue treatment for Helicobacter pylori infection, so that there is a considerable amount of evidence to assess the safety of furazolidone-containing regimen.

- Effects of duration and dose on the safety of furazolidone are also analysed.

- The reporting of this review strictly follows the PRISMA (Preferred Reporting Items for Systematic Reviews and Meta-Analyses) statement. For the main results, sample size is measured by trial sequential analysis, and quality of evidence is graded according to the GRADE (grading of recommendations assessment, development and evaluation) approach.

- Limitations include most studies being openlabelled and lack of data from developed countries, which restricts the generalisability of study findings.

$89 \%$ cases of non-cardia gastric cancer, which accounts for $78 \%$ gastric cancers, are attributed to $H$. pylori. ${ }^{2}{ }^{3}$ Early detection and eradication of $H$. pylori can prevent the progression of gastric atrophy and reduce relative risks (RRs) for developing gastric cancers. $^{45}$

Facing the yearly increasing antibiotic resistance of $H$. pylori worldwide, ${ }^{6}$ traditional antibiotic-containing therapy is no longer reliable to achieve satisfying eradication rate. ${ }^{7}$ Additionally, failure of first-line therapy exacerbates the difficulty for rescue treatment with significantly increased clarithromycin and metronidazole resistance. ${ }^{8}$ Therefore, it's imperative to introduce new antibiotics with low drug resistance for the current regimen. As resistance of $H$. pylori to furazolidone remains below 5\% in Asia and South America, ${ }^{9}$ it may be a key component in treatment success, especially in regions with high antibiotic resistance.

Furazolidone is a synthetic nitrofuran derivative with a broad antibacterial and antiprotozoal spectrum to treat gastrointestinal 
tract infections. ${ }^{10}$ It's well-absorbed by oral administration, and was first used to treat $H$. pylori infection in 1985. ${ }^{11}$ Few genetic mutations have been identified in $H$. pylori for its resistance, and rare cross-resistance was observed between furazolidone and other antibiotics, ${ }^{10}$ 1213 indicating it could be a good candidate for $H$. pylori eradication. However, the availability of furazolidone was restricted in developed countries for potential genotoxic and carcinogenetic effects in animal experiments, ${ }^{14-16}$ but further research failed to provide any fundamental clinical evidence neither from case reports nor epidemiological studies.

Meanwhile, it's still available and widely used in developing countries owing to its good cost-effectiveness. Plenty of randomised controlled trials (RCTs) have confirmed the high efficacy of furazolidone in both initial and rescue treatment. ${ }^{17} \mathrm{~A}$ recent meta-analysis showed furazolidone was more effective than other antibiotics in the first-line quadruple therapy, with a pooled eradication rate exceeding $90 \%$ for per-protocol analysis. But up to now, there is still no definite answer to the safety of furazolidone in $H$. pylori eradication.

Herein, we systematically reviewed relevant RCTs up to date to assess the safety and compliance of furazolidone versus other antibiotic containing regimen for $H$. pylori eradication, and further evaluated its safety in variable durations and dose schemes.

\section{METHOD}

This meta-analysis was reported according to PRISMA (Preferred Reporting Items for Systematic Reviews and Meta-Analyses) statement, ${ }^{18}$ and registered on PROSPERO (international prospective register of systematic reviews) with the number: CRD42019137247.

\section{Search strategy}

Systematic literature search was conducted in PubMed, Embase, Cochrane Library, Web of Science and Scopus databases from inception to June 2019. A combination of $\mathrm{MeSH}$ and free terms was used to identify relevant clinical trials, including Furazolidine (with variations: Nifurazolidone, Furoxone or Furazol) and Helicobacter (with variations: Helicobacter pylori and Campylobacter pylori), with filters of RCTs applied to all the searching results (online supplemental appendix A). Clinicaltrials.gov, GreyNet, BIOSIS Previews, OCLC FirstSearch databases were also searched for unpublished trials and consensus reports with the same strategy. Then, we emailed authors to verify the results of retrieved studies, and manually checked reference lists of reviews, letters and included articles to identify any other relevant studies.

\section{Inclusion and exclusion criteria}

Only RCTs published in English or Chinese were eligible. The inclusion criteria were as follows: (1) H. pylori infection was confirmed by at least one standard detection method, including urea breath test, rapid urease test, histology, culture or faecal antigen testing. (2) Studies included at least two arms of treatments comparing furazolidone with non-furazolidone-containing regimen, different treatment durations or various doses of furazolidone. (3) Studies compared furazolidone-containing therapy with placebo or proton pump inhibitor were also included. (4) Incidence of total adverse events (AEs) and serious AEs should be monitored and available for each study arm. Exclusion criteria included: (1) Studies that enrolled paediatric patients or patients with specific underlying disease. (2) Studies that used a daily dose of furazolidone over $400 \mathrm{mg}$ (the highest recommended daily dose for adults). (3) Studies that compared different forms of furazolidone regimens (eg, quadruple versus triple therapy). (4) Studies that changed the dose, duration of drugs other than furazolidone or assessed additional interventions. (5) Studies with treatment duration less than 5 days or over 14 days. (6) Studies with incomplete safety data after contacting authors, including a blurry description of safety outcomes, failing to provide a separate incidence of total AEs for each study arm and lack of serious AEs recording.

\section{Study selection}

After removal of duplicates, two reviewers independently screened all the abstracts following the selection criteria to identify relevant studies. When a decision could not be made solely based on the abstract, full text was further reviewed to assess the inclusion. Any discrepancies between the two reviewers were resolved by discussion with a third reviewer.

\section{Data extraction}

Two reviewers separately used a standardised, electronic data collection form to extract all the relevant data from included studies. Primary outcomes were the incidence of total AEs and serious AEs. We adopted a definition of serious AEs from the International Council for Harmonisation ( $\mathrm{ICH}$ ) harmonised tripartite guideline E2A. ${ }^{19}$ Serious AEs were defined as life-threatening events requiring hospitalisation or prolonged existing hospitalisation, or resulting in persistent disability and even death. When available, the severity of AEs was also extracted. Severe AEs were defined as significant limitations to daily activity and sometimes even led to drug withdrawal. ${ }^{20}$ Secondary outcomes were the incidence of individual adverse symptoms, the incidence of AE-related treatment discontinuation and compliance. Types of individual adverse symptoms were defined by preferred terms from the Medical Dictionary for Regulatory Activities V.19.0. ${ }^{21}$ The list of preferred terms included: gastrointestinal disorders (nausea, vomiting, diarrhoea, constipation, dyspepsia, abdominal pain, abdominal discomfort and flatulence), nervous system disorders (dizziness, somnolence, dysgeusia and headache), skin and subcutaneous tissue disorders (rash and pruritus), psychiatric disorders (anorexia and insomnia), general disorders (fatigue, fever and chills) and other specific symptoms. For analysis of 
compliance, only patients taking at least one dose of drug were included, and the acceptable compliance level was defined as $>80 \%$ for general acknowledgement. Besides, additional drug interventions, collecting methods of AEs, dose and duration of regimens were extracted for further analysis. Demographic characteristics such as age, country and baseline disease status were also extracted. Any observed data differences between the two collecting forms were checked against original texts and then examined by another reviewer to minimise human errors.

\section{Risk of bias assessment}

Two reviewers independently evaluated the methodological quality of RCTs using the first version of Cochrane Collaboration Risk of Bias tool ${ }^{22}$ with RevMan software V.5.3.5 (Nordic Cochrane Centre, Copenhagen, Denmark, 2014). When differences could not be solved by group discussions, a third reviewer was invited to make the final decision.

\section{Data synthesis, analysis and grading of evidence}

Meta-analysis was conducted using R software V.3.6.0 (R Foundation for Statistical Computing, Vienna, Austria, 2019). For pooled estimates of dichotomous outcomes, RR and 95\% CI were calculated and synthesised by the Mantel-Haenszel approach. Significant $p$ value was set at 0.05 . Random effects model was preferentially applied for conservative evaluation of treatment effect size across studies. Statistical heterogeneity was assessed using both the $\mathrm{Q}$ test and $I^{2}$ statistic. A p value $<0.1$ for the $Q$ test or $I^{2}$ value $>50 \%$ indicated significant heterogeneity. ${ }^{23}$ Then, subgroup analysis would be done to identify the possible causes. Subgroup categories included dose of furazolidone, quadruple or triple forms of regimens, country of patients, prompted collection (collecting AEs with active return visit call or interview) or passive collection of AEs (collecting AEs with written questionnaires or report cards). Risk of publication bias was assessed by funnel plots and quantified by the Egger's linear regression test ${ }^{24}$ and the Begg's rank correlation test. ${ }^{25}$ Trim and fill method was applied to revise existing publication bias. ${ }^{26}$ Sensitivity analysis was performed by continuously excluding every single study in the pooled estimate, and recalculating the RR with remaining studies. The synthesised result would be considered unreliable if any obvious alterations occurred after exclusion.

For the incidence of total AEs between furazolidone and non-furazolidone-containing regimen, trial sequential analysis (TSA) was conducted to estimate the required information size using TSA viewer software V.0.9.5.10 (Copenhagen trial unit, Copenhagen, Denmark, 2016). ${ }^{27}{ }^{28}$ Besides, two investigators independently graded the quality of evidence at outcome level, following the grading of recommendations assessment, development and evaluation (GRADE) approach. ${ }^{29}$

\section{RESULTS}

\section{Search results and study characteristics}

As shown in figure 1, a total of 2039 records were identified, of which 100 records were further assessed for eligibility. Finally, 26 articles met the selection criteria, enrolled in the meta-analysis and were further classified into three groups for different study aims. The consistency of study selection was good between two reviewers ( $\kappa$ statistic $=0.83$ ). Four of 26 articles involved two comparisons. Data from these articles were separately analysed and relevant sources were listed as a single study in each comparison group.

Fourteen studies compared furazolidone with nonfurazolidone-containing regimen. The pooled intentionto-treat eradication rate was significantly higher in furazolidone containing ones (RR 1.06, 95\% CI 1.01 to 1.12 , online supplemental figure S1a). Similar superiority was also found in the per-protocol analysis (RR 1.05, $95 \%$ CI 1.00 to 1.10 , online supplemental figure S1b). Twelve studies ${ }^{30}$ evaluated the safety of furazolidone with different treatment durations, and four studies assessed variable doses. Prolonged duration to 14 days and higher daily dose significantly elevated the treatment efficacy (RR $1.05,95 \%$ CI 1.02 to 1.08 ; RR 1.23, 95\% CI 1.07 to 1.43). The main characteristics of above studies are summarised in table 1.

\section{Risk of bias across the studies}

Five studies were open-labelled trials, leading to high risk for performance bias. Twenty-one studies ${ }^{30}$ used return visits or telephone interviews to promptly collect AEs without blinding to treatment allocation, which caused a high risk in detection bias. One study partially reported moderate and severe AEs, resulting in a high risk in reporting bias. Other biasses were low or unclear in most studies (online supplemental figure S2).

\section{Furazolidone versus non-furazolidone containing regimen Overall safety outcomes}

Fourteen studies involving 2540 patients showed furazolidone group and non-furazolidone group had a similar risk of total AEs (RR 1.04, 95\% CI 0.89 to 1.21, figure 2A), with a pooled incidence rate of $19.33 \%(236 / 1,221)$ and $17.59 \%$ (232/1,319), respectively. Subgroup analysis by dose, duration and quadruple or triple forms of regimens also found no significant difference. Only two serious AEs were reported in furazolidone group $(0.16 \%, 2 / 1,221)$. Both the patients received furazolidone and amoxicillin quadruple therapy, and were hospitalised for suspicion of allergy. No serious AEs were reported in non-furazolidone group. Five studies reported the incidence of severe AEs. The pooled incidence rates in the two groups were $5.82 \%(22 / 378)$ and $2.74 \%(10 / 365)$, with no significant 


\begin{tabular}{|l|}
1906 Records identified through database search \\
184 PubMed \\
524 Embase \\
68 Cochrane Library \\
176 Web of Science core collection \\
772 Scopus \\
182 Other source of database
\end{tabular}

133 Additional articles identified from reviews and manual search

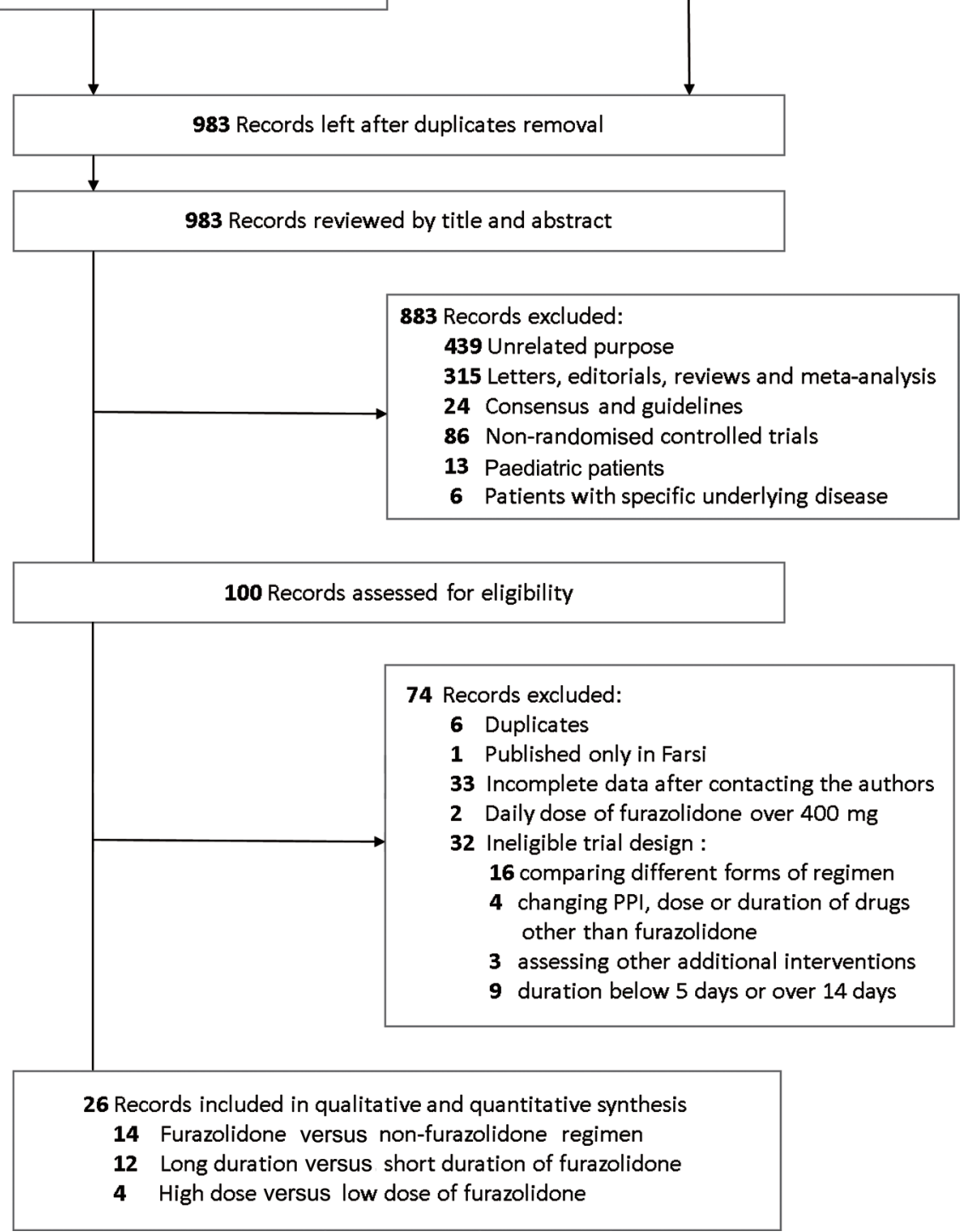

Figure 1 Flow chart for study selection. PPI, proton pump inhibitor.

increased risk detected (RR 1.81, 95\% CI 0.91 to 3.60, figure $2 B$ ).

TSA analysis was performed for the incidence of total AEs between the two groups. Although the pooled population did not reach the estimated sample size, cumulative $\mathrm{Z}$ curve surpassed the inner futility line, indicating no significant difference would be detected even with an increased number of patients (online supplemental figure S3).

Individual adverse symptoms and compliance

Twelve studies provided detailed individual adverse symptoms, of which nausea and dizziness were commonly reported with a pooled incidence of $7.72 \%$
(74/958) vs $9.18 \%(90 / 980), 6.95 \%(56 / 806)$ vs $6.29 \%$ $(52 / 827)$, respectively. No significant differences were found (table 2) similarly for the results of abdominal pain, diarrhoea, vomiting, headache, fever, skin rash and anorexia.

Subgroup analysis by passive reports collection even found a lower RR of nausea in furazolidone group (RR $0.42,95 \%$ CI 0.22 to 0.79 , online supplemental figure S4). However, nine studies found a higher risk of dysgeusia in non-furazolidone group (RR $0.57,95 \%$ CI 0.35 to 0.93 , table 2 ) .

Incidence of AE-related treatment discontinuation was similar in furazolidone $(3.22 \%(19 / 590))$ versus nonfurazolidone group $(2.25 \%(13 / 577))$, with a RR of 1.30 


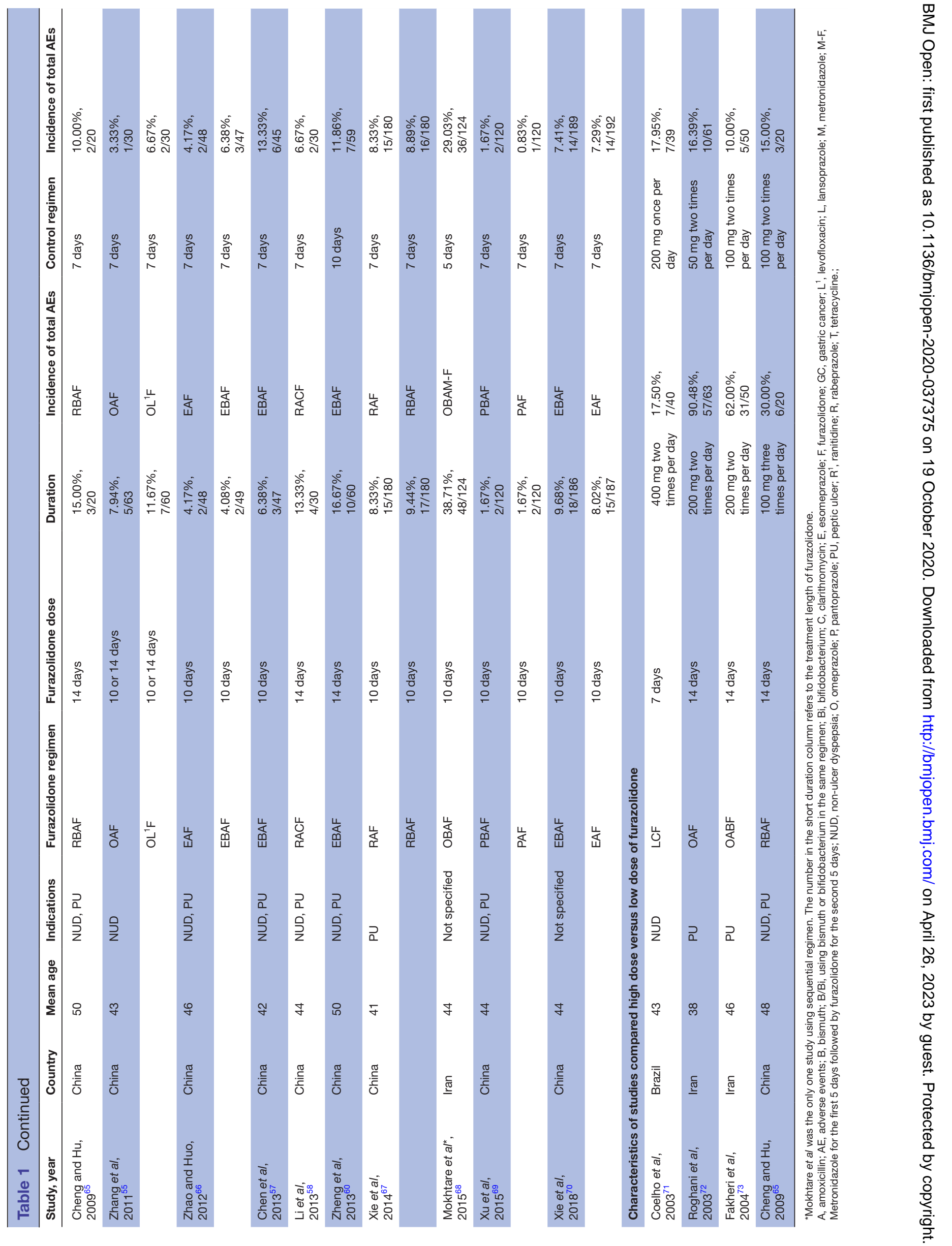


a

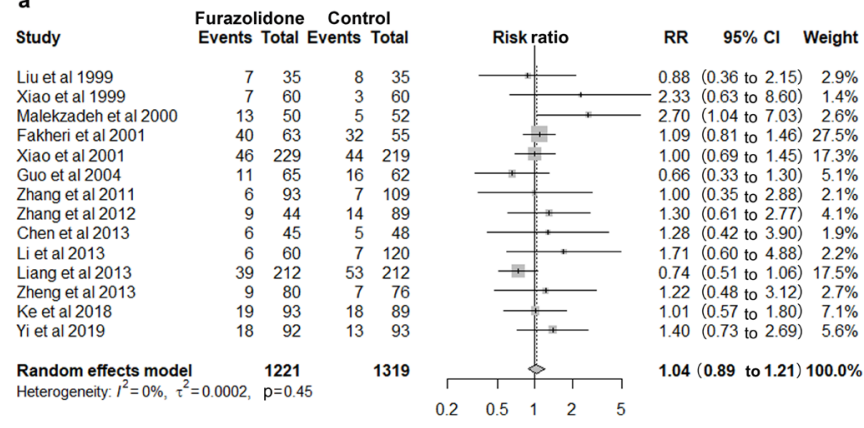

b

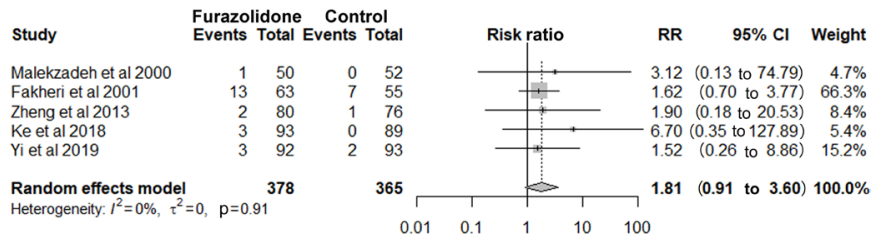

Figure 2 Furazolidone versus non-furazolidone-containing regimen: (a) Incidence of total adverse events. (b) Incidence of severe adverse events. RR, relative risk.

(95\% CI 0.65 to 2.63, table 2). Patients' compliance was almost the same between two groups. (96.94\% vs $96.67 \%$, RR $1.00,95 \%$ CI 0.99 to 1.01, table 2). Low heterogeneities were detected in these analysis.

\section{Long duration versus short duration of furazolidone}

Overall safety outcomes

Twelve studies involving 3139 patients found a higher risk of total AEs in the long-duration group (RR 1.33, 95\% CI 1.09 to 1.61 ), which was dose-related and became nonsignificant with a daily dose of $200 \mathrm{mg}$ (RR $1.27,95 \%$ CI 0.92 to 1.76 , figure 3 ). The majority of AEs were still welltolerated, no serious AEs occurred in either group and no increased risk of severe AEs was observed (RR 1.04, $95 \%$ CI 0.38 to 2.86 , table 2 ).

\section{Individual adverse symptoms and compliance}

Nine studies were included for analysis of individual adverse symptoms. ${ }^{30}$ No increased risk was observed for nausea, dizziness, vomiting, diarrhoea or fatigue. Risk of AE-related treatment discontinuation and patients' compliance were also similar between the two groups. The heterogeneities were low for the above comparisons (table 2).

\section{High dose versus low dose of furazolidone}

\section{Overall safety outcomes}

Four studies with a total of 343 patients found an increased risk of total AEs in high daily dose of furazolidone (RR $3.04,95 \%$ CI 1.28 to 7.22 , figure 4 A). Subgroup analysis showed similar result in a 14-day regimen with low heterogeneity (RR 4.87, 95\% CI 2.89 to 8.18, figure 4A). Similarly, an elevated risk of severe AEs was observed in high-dose group (RR 3.74, 95\% CI 1.29 to 10.86, figure 4B), but none of them was classified as serious AEs among all the involved patients.

\section{Individual adverse symptoms and compliance}

Data from three studies showed an obvious increased risk of nausea and dizziness in high dose versus low dose of furazolidone (RR 4.63, 95\% CI 1.49 to 14.40 ; RR 12.28, $95 \%$ CI 2.95 to 51.07, respectively, table 2), but risk was similar for diarrhoea and headache. For analysis of compliance, a high heterogeneity arose from Roghani $e t$ al, 2003, which used a four-fold dose of furazolidone in the high-dose group compared with control. Subgroup analysis showed compliance was not compromised in a higher dose regimen. Risk of treatment discontinuation was also similar regardless of dosage change (table 2).

\section{Grading of evidence}

All the involved RCTs had serious study limitations for lack of allocation concealments or blinding to the treatment arms. Accordingly, the certainty of evidence was downgraded to a moderate level for most conclusions. In high dose versus low dose of furazolidone, the quality of evidence was rated low for a wide CI (table 2).

\section{Publication bias}

No publication bias were detected by the Egger's and Begg's test for the main outcomes (table 2).

\section{Sensitivity analysis}

For incidence of dysgeusia between furazolidone and non-furazolidone-containing regimen, the pooled estimates obviously shifted to a no significant level after excluding Fakheri et al, 2001 (online supplemental figure S5). For analysis between variable doses of furazolidone, the synthetic results were unstable for incidence of total AEs, severe AEs and nausea. But incidence of total AEs and nausea became robust to sensitivity analysis after additionally included Hosseini et $a l,{ }^{31} 2014$, which compared a daily dose of furazolidone in $600 \mathrm{mg}$ versus $400 \mathrm{mg}$ (online supplemental figure S6).

\section{DISCUSSION}

In this meta-analysis, data of 2540 patients from 14 RCTs showed that furazolidone-containing regimen had no increased risks of total AEs or severe AEs, while maintaining higher efficacy compared with non-furazolidonecontaining regimen. Only two serious AEs were reported in furazolidone group. The majority of AEs were welltolerated with a low incidence of discontinuation and excellent compliance $(>95 \%)$ to the treatment. ${ }^{32}$

Compared with other antibiotic regimens, furazolidonecontaining regimen had superior efficacy with a similar risk of total AEs, irrespective of altered daily dose, duration and regimen forms. These results were consistent with findings in a recent meta-analysis, in which no increased risks of total AEs and severe AEs were found in seven RCTs comparing furazolidone with other antibioticcontaining regimens. The RR was $1.01(95 \% \mathrm{CI} 0.91$ to 1.11) for total AEs and 1.70 (95\% CI 0.84 to 3.47) for severe AEs. But these results were partially collected for 


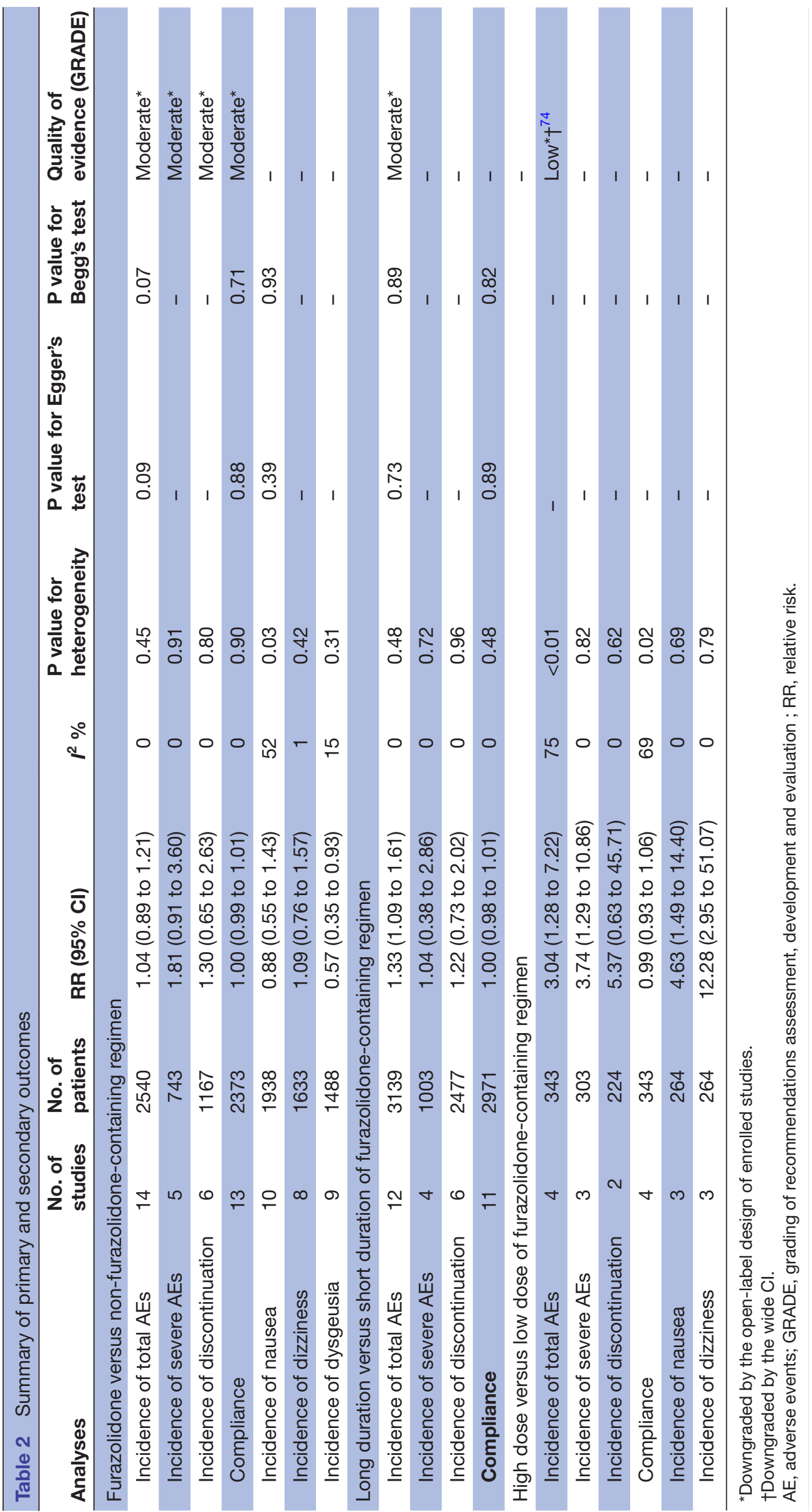




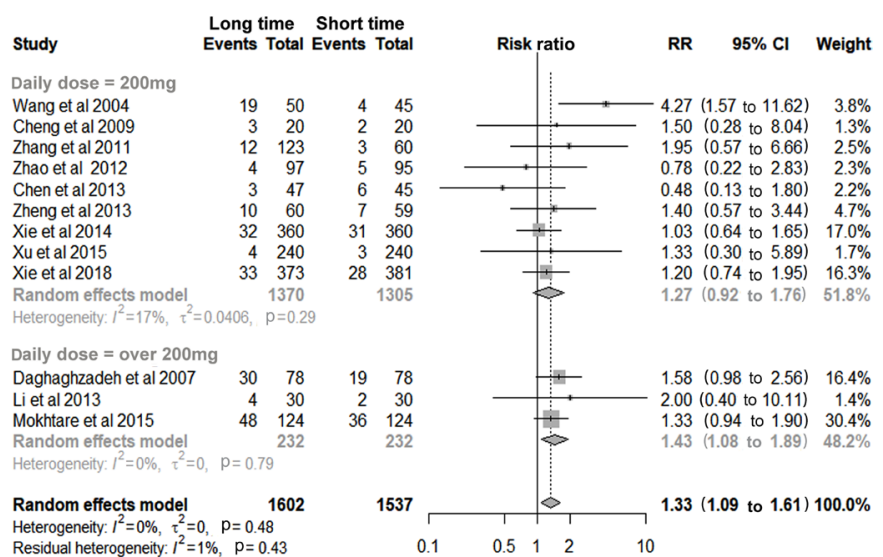

Figure 3 Long duration versus short duration of furazolidone for incidence of total adverse events.

patients with naïve infection. We additionally included patients receiving rescue treatments, designed a research strategy specialised for safety outcomes and further verified these findings. However, the quality of evidence was moderate, as most RCTs were poorly designed without allocation concealment and blinding method.

For analysis of individual adverse symptoms, no increased risks of nausea, vomiting, abdominal pain, diarrhoea, headache, fever, skin rash or anorexia were detected in patients receiving furazolidone. While, patients receiving other antibiotics were easy to have dysgeusia, which was probably caused by the wide clarithromycin use in 11 of 14 studies. But these results should be taken seriously, as sensitivity analysis detected significant alterations in the pooled estimates after excluding certain study. More evidence is required to draw a confirmed conclusion.

Additionally, we assessed the safety outcomes in variable duration and dose of furazolidone. Among patients who received a daily dose of $200 \mathrm{mg}$, no increased risk of total AEs was detected even with extended treatment duration to 14 days. When given a high daily dose of furazolidone

\section{a}

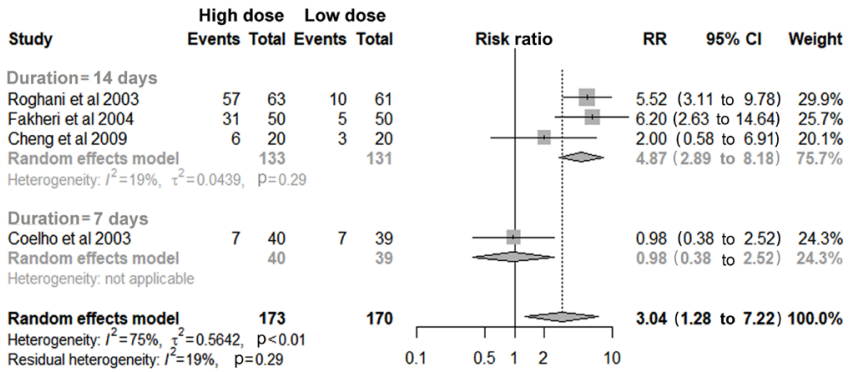

b

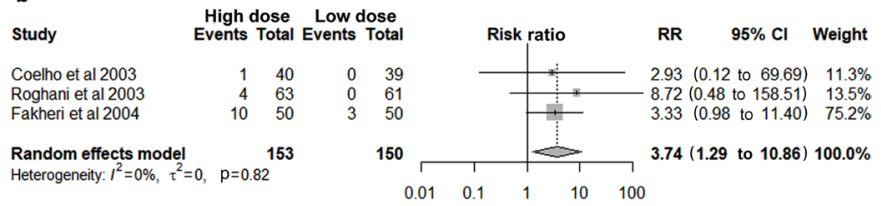

Figure 4 High dose versus low dose of furazolidone: (a) Incidence of total adverse events. (b) Incidence of severe adverse events. $\mathrm{RR}$, relative risk. ranging from $300 \mathrm{mg}$ to $400 \mathrm{mg}$, patients had an obvious higher risk of total AEs and severe AEs compared with the low-dose regimen. Meanwhile, the incidence of nausea and dizziness also became more frequent, which was similar with results reported by Zhuge $e t a l .{ }^{33}$ These findings suggested that prescription of furazolidone should be started with a minimum necessary dose of $100 \mathrm{mg}$ twice daily to avoid potential severe AEs. If a low-dose regimen fails to achieve expected therapeutic efficacy, extending the duration of furazolidone should be first considered rather than increasing the daily dose.

Concerns over furazolidone related irreversible AEs restricted its availability in developed countries, but up to now, no supporting clinical evidence has been reported. The International Agency for Research on Cancer (IARC) classified furazolidone as a category 3 agent with unclassifiable carcinogenicity, while metronidazole was classified as a category 2B agent with possible carcinogenicity to humans. ${ }^{34}$ Some researchers pointed out there might be some misunderstanding of furazolidone. ${ }^{35}$ Currently, the recommended $H$. pylori eradication schedules were 10 to 14 days. Considering the resistance to furazolidone is still rare worldwide, the benefits of short-term clinical use could easily overweigh the possible but low risk.

China has a long history use of furazolidone to cure ulcers even before the discovery of $H$. pylori. Our results showed that among 4505 patients receiving furazolidone-containing regimen in 26 trials, only two cases of serious AEs were reported with a rare incidence rate of $0.04 \%$, which indicated the adverse reactions of furazolidone might be exaggerated in previous reviews. Both the patients received furazolidone and amoxicillin quadruple therapy, and were hospitalised for severe skin rash, flushing and abdominal colic. As allergy to penicillin is commonly reported in $10 \%$ of the population, hypersensitivity reactions to amoxicillin could not be ruled out. ${ }^{36}$ Three patients reported numbness of limbs with suspicion of peripheral neuritis, but symptoms relieved spontaneously after stopping treatment and supplements of vitamin B1 and B6. Other severe adverse symptoms mostly disappeared after drug withdrawal without additional treatments (online supplemental table S1).

The occasionally occurring severe AEs were most likely related to the monoamine oxidase inhibitory properties of furazolidone. ${ }^{37}$ One of its major metabolite, amino-2oxazolidone, can non-selectively inhibit the monoamine oxidase activity, interact with metabolism of tyramine and cause dose-dependent gastrointestinal and nervous system disorders. ${ }^{38}$ Notably, two specific AEs were reported in previous studies. One was disulfiram-like reaction to alcohol and the other was haemolytic anaemia in glucose-6-phosphate dehydrogenase (G-6-PD) deficient patients. ${ }^{39}$ Thus, it should not be given concurrently to individuals already taking other monoamine oxidase inhibitors or antidepressants, or having decreased G-6-PD activity. Alcohol should also be avoided when furazolidone is being used. 
Apart from the combination with antacids for $H$. pylori eradication, furazolidone was widely used alone for the treatment of traveller's diarrhoea, typhoid fever, cholera and salmonella infections. The most commonly reported AEs were gastrointestinal distress, dizziness, somnolence, headaches and general malaise. ${ }^{40}$ In a review that quantified 191 studies, the frequency of AEs to furazolidone was $8.3 \%(864 / 10443)$ in gastrointestinal infections. Most adverse symptoms were mild, with an incidence below $1 \%$, and no drug-induced death recorded. One RCT compared furazolidone (100 mg once a day for 5 days) with ampicillin among 94 patients with traveller's diarrhoea. Only one patient receiving furazolidone dropped out due to local skin rash. ${ }^{41}$ Another RCT assessed different doses of furazolidone in 57 children with cholera. No drug-related discontinuation of treatment occurred. ${ }^{42}$ These data further confirm the safety of furazolidone as a single agent in treating a infectious disease.

We first evaluated the safety of furazolidonecontaining regimen as a primary outcome and pointed out the increased risk of AEs was mainly attributed to the high daily dose of furazolidone. In previous metaanalyses, ${ }^{43} 44$ safety of furazolidone regimen was only assessed as a secondary outcome, and relevant data was partially collected from RCTs eligible for efficacy analysis. Under this condition, selection bias was inevitable for incomplete data retrieval. To overcome these drawbacks, we first developed a search strategy specialised for safety evaluation, additionally included both initial and rescue treatments, and updated search scopes from 2016 to June 2019. Besides, we analysed the safety of furazoidone in variable doses and durations schemes, and first concluded a daily dose of $200 \mathrm{mg}$ is safe for current 14-day eradication regimen.

This meta-analysis did have several limitations. First, most included studies did not mention allocation concealment and blinding method in the study design, which led to high detection bias of AEs reporting. Therefore, more high-quality evidence from doubleblind RCTs is warranted to assess the safety outcomes accurately. Second, as the completion of treatment was around 6 to 8 weeks, incidence of delayed adverse reactions cannot be evaluated with limited follow-up duration. Finally, current conclusions are mainly based on clinical data from Asian people and developing countries. Available safety data in Western people are all from small pilot studies, and no serious AEs was observed. ${ }^{45-50}$ More large-scale clinical trials are needed to assess the effectiveness and safety of furazolidonecontaining regimen in developed countries.

In conclusion, furazolidone has similar risk of AEs as non-furazolidone-containing regimens, while maintaining good efficacy and high compliance. The majority of AEs are mild-to-moderate with a low occurrence of treatment discontinuation and excellent compliance of patients, which is not compromised by increased dose and duration of furazolidone. Higher incidence of total AEs and severe AEs for furazolidone are mainly attributed to increased dose rather than prolonged duration. A low daily dose of $200 \mathrm{mg}$ is safe and welltolerated for 14-day regimen, which should be recommended for $H$. pylori infection.

Acknowledgements We are grateful to Rui Zhao and Qing-fang Zhang for providing the original data to support our research.

Collaborators Not applied.

Contributors XZ and CJ designed the study and wrote the manuscript. CJ, JL and $Y L$ collected the data and evaluated the quality of evidence. CJ analysed the data. $\mathrm{CG}, \mathrm{JQ}$ and YZ helped with data interpretation and gave a critical review of the manuscript. All the authors have approved the final version of the manuscript.

Funding This research was supported by the National Natural Science Foundation of China (81 770538 and 81570485), the Key Research and Development Program of Shandong Province (2017CXGC1215).

Competing interests None declared.

Patient and public involvement Patients and/or the public were not involved in the design, or conduct, or reporting, or dissemination plans of this research.

Patient consent for publication Not required.

Provenance and peer review Not commissioned; externally peer-reviewed.

Data availability statement Data are available upon reasonable request.

Open access This is an open access article distributed in accordance with the Creative Commons Attribution Non Commercial (CC BY-NC 4.0) license, which permits others to distribute, remix, adapt, build upon this work non-commercially, and license their derivative works on different terms, provided the original work is properly cited, appropriate credit is given, any changes made indicated, and the use is non-commercial. See: http://creativecommons.org/licenses/by-nc/4.0/.

ORCID iD

Xiuli Zuo http://orcid.org/0000-0001-9556-8771

\section{REFERENCES}

1 Zamani M, Ebrahimtabar F, Zamani V, et al. Systematic review with meta-analysis: the worldwide prevalence of Helicobacter pylori infection. Aliment Pharmacol Ther 2018;47:868-76.

2 Peek RM, Blaser MJ. Helicobacter pylori and gastrointestinal tract adenocarcinomas. Nat Rev Cancer 2002;2:28-37.

3 Plummer M, Franceschi S, Vignat J, et al. Global burden of gastric cancer attributable to Helicobacter pylori. Int J Cancer 2015;136:487-90.

4 Ford AC, Forman D, Hunt R, et al. Helicobacter pylori eradication for the prevention of gastric neoplasia. Cochrane Database Syst Rev 2015:CD005583.

5 Lee Y-C, Chiang T-H, Chou C-K, et al. Association between Helicobacter pylori eradication and gastric cancer incidence: a systematic review and meta-analysis. Gastroenterology 2016;150:1113-24.

6 Thung I, Aramin H, Vavinskaya V, et al. Review article: the global emergence of Helicobacter pylori antibiotic resistance. Aliment Pharmacol Ther 2016;43:514-33.

7 Graham DY, Fischbach L. Helicobacter pylori treatment in the era of increasing antibiotic resistance. Gut 2010;59:1143-53.

8 Savoldi A, Carrara E, Graham DY, et al. Prevalence of Antibiotic Resistance in Helicobacter pylori: A Systematic Review and Metaanalysis in World Health Organization Regions. Gastroenterology 2018;155:1372-82. e17.

9 Zamani M, Rahbar A, Shokri-Shirvani J. Resistance of Helicobacter pylori to furazolidone and levofloxacin: A viewpoint. World $\mathrm{J}$ Gastroenterol 2017;23:6920-2.

10 Grayson M. Lindsay M, Crowe S, et al. Kucers' the use of antibiotics. 7 th ed. Boca Raton, Florida, America: CRC press, 2017: 3187-94.

11 Zheng ZT, Wang ZY, Chu YX, et al. Double-Blind short-term trial of furazolidone in peptic ulcer. Lancet 1985;1:1048-9.

12 Kwon DH, Lee M, Kim JJ, et al. Furazolidone- and nitrofurantoinresistant Helicobacter pylori: prevalence and role of genes involved in metronidazole resistance. Antimicrob Agents Chemother 2001;45:306-8. 
13 Su Z, Xu H, Zhang C, et al. Mutations in Helicobacter pylori porD and oorD genes may contribute to furazolidone resistance. Croat Med $\mathrm{J}$ 2006;47:410-5.

14 Graham DY, Lu H. Furazolidone in Helicobacter pylori therapy: misunderstood and often unfairly maligned drug told in a story of French bread. Saudi J Gastroenterol 2012;18:1-2.

15 Gajewska J, Szczypka M, Tudek B, et al. Studies on the effect of ascorbic acid and selenium on the genotoxicity of nitrofurans: nitrofurazone and furazolidone. Mutat Res 1990;232:191-7.

16 Tatsuta M, lishi H, Baba M, et al. Attenuating effect of the monoamine oxidase inhibitor furazolidone on the anti-carcinogenetic effect of cysteamine on gastric carcinogenesis induced by $\mathrm{N}$ methyl-N'-nitro-N-nitrosoguanidine in Wistar rats. Int $J$ Cancer 1991;48:605-8.

17 Malekzadeh R, Ansari R, Vahedi $\mathrm{H}$, et al. Furazolidone versus metronidazole in quadruple therapy for eradication of Helicobacter pylori in duodenal ulcer disease. Aliment Pharmacol Ther 2000;14:299-303.

18 Liberati A, Altman DG, Tetzlaff J, et al. The PRISMA statement for reporting systematic reviews and meta-analyses of studies that evaluate healthcare interventions: explanation and elaboration. $B M J$ 2009;339:b2700.

$19 \mathrm{ICH}$. Clinical safety data management: definitions and standards for Expedited reporting E2A 1994.

20 Zheng XL, Xu L. Comparison of 10-day and 14-day furazolidonecontaining quadruple salvage therapies for Helicobacter pylori eradication. Chinese Journal of New Drugs 2013;22:2786-8.

21 Medical dictionary for regulatory activities. Available: https://www. meddra.org [Accessed 21 July 2018].

22 Higgins JPT, Altman DG, Gøtzsche PC, et al. The Cochrane collaboration's tool for assessing risk of bias in randomised trials BMJ 2011;343:d5928.

23 Higgins JPT, Thompson SG. Quantifying heterogeneity in a metaanalysis. Stat Med 2002;21:1539-58.

24 Egger M, Davey Smith G, Schneider M, et al. Bias in meta-analysis detected by a simple, graphical test. BMJ 1997;315:629-34.

25 Begg CB, Mazumdar M. Operating characteristics of a RANK correlation test for publication bias. Biometrics 1994;50:1088-101.

26 Peters JL, Sutton AJ, Jones DR, et al. Performance of the TRIM and fill method in the presence of publication bias and between-study heterogeneity. Stat Med 2007;26:4544-62.

27 Brok J, Thorlund K, Gluud C, et al. Trial sequential analysis reveals insufficient information size and potentially false positive results in many meta-analyses. J Clin Epidemiol 2008;61:763-9.

28 Wetterslev J, Thorlund K, Brok J, et al. Trial sequential analysis may establish when firm evidence is reached in cumulative meta-analysis. $J$ Clin Epidemiol 2008:61:64-75.

29 Guyatt G, Oxman AD, Akl EA, et al. Grade guidelines: 1. IntroductionGRADE evidence profiles and summary of findings tables. J Clin Epidemiol 2011;64:383-94.

30 Wang F, Wang X-yan, Jia Y, et al. [Five-day rabeprazole-based triple therapy for helicobacter pylori infection]. Zhong Nan Da Xue Xue Bao Yi Xue Ban 2004;29:589-91.

31 Hosseini V, Mokhtare M, Gholami M, et al. A comparison between moderate- and high-dose furazolidone in triple regimens for Helicobacterpylori eradication in Iran. Middle East J Dig Dis 2014;6:195-202.

32 Graham DY, Lu H, Yamaoka Y. A report card to grade Helicobacter pylori therapy. Helicobacter 2007;12:275-8.

33 Zhuge L, Wang Y, Wu S, et al. Furazolidone treatment for Helicobacter Pylori infection: A systematic review and meta-analysis. Helicobacter 2018;23:e12468.

34 WHO. IARC monographs on the identification of carcinogenic hazards to humansWorld health organization. Available: https:// monographs.iarc.fr/list-of-classifications/ [Accessed 2020-1-20]

35 Mohammadi M, Attaran B, Malekzadeh R, et al. Furazolidone, an underutilized drug for $\mathrm{H}$. pylori eradication: lessons from Iran. Dig Dis Sci 2017;62:1890-6.

36 Shenoy ES, Macy E, Rowe T, et al. Evaluation and management of penicillin allergy: a review. JAMA 2019;321:188-99.

37 Palm D, Magnus $\mathrm{U}$, Grobecker $\mathrm{H}$, et al. [Inhibition of monoamine oxidase by bacteriostatically active nitrofuran derivatives]. Naunyn Schmiedebergs Arch Exp Pathol Pharmakol 1967;256:281-300.

38 Karamanakos PN. Furazolidone and serotonin syndrome: is there any association? Clinics 2008;63:553-4.

39 Altamirano A, Bondani A. Adverse reactions to furazolidone and other drugs. A comparative review. Scand J Gastroenterol Suppl 1989;169:70-80.

40 Phillips KF, Hailey FJ. Furazolidone for treatment of diarrhoeal disease. Trop Doct 1987;17:89-91.
41 DuPont HL, Ericsson CD, Galindo E, et al. Furazolidone versus ampicillin in the treatment of traveler's diarrhea. Antimicrob Agents Chemother 1984;26:160-3.

42 Rabbani GH, Butler T, Shahrier M, et al. Efficacy of a single dose of furazolidone for treatment of cholera in children. Antimicrob Agents Chemother 1991;35:1864-7.

43 XJ Q, Shi BX, Jiang Y. Furazolidone-based firstline therapy for Helicobacter pylori infection in China: a meta-analysis. World Chinese Journal of Digestology 2016;24:4484-90.

44 Buzás GM, Józan J. Nitrofuran-based regimens for the eradication of Helicobacter pylori infection. J Gastroenterol Hepatol 2007;22:1571-81.

45 Van Zwet AA, Thijs JC, van der Wouden EJ, et al. Low cure rate of Helicobacter pylori infection with omeprazole and furazolidone dual therapy for one week. Aliment Pharmacol Ther 1997;11:533-5.

46 Graham DY, Osato MS, Hoffman J, et al. Furazolidone combination therapies for Helicobacter pylori infection in the United States. Aliment Pharmacol Ther 2000;14:211-5.

47 Isakov V, Domareva I, Koudryavtseva L, et al. Furazolidone-based triple 'rescue therapy' vs. quadruple 'rescue therapy' for the eradication of Helicobacter pylori resistant to metronidazole. Aliment Pharmacol Ther 2002;16:1277-82.

48 Treiber G, Ammon S, Malfertheiner P, et al. Impact of furazolidonebased quadruple therapy for eradication of Helicobacter pylori after previous treatment failures. Helicobacter 2002;7:225-31.

49 Qasim A, Sebastian S, Thornton O, et al. Rifabutin- and furazolidonebased Helicobacter pylori eradication therapies after failure of standard first- and second-line eradication attempts in dyspepsia patients. Aliment Pharmacol Ther 2005;21:91-6.

50 Tay CY, Windsor HM, Thirriot F, et al. Helicobacter pylori eradication in Western Australia using novel quadruple therapy combinations. Aliment Pharmacol Ther 2012;36:1076-83.

51 Liu WZ, Xiao SD, Shi Y, et al. Furazolidone-containing short-term triple therapies are effective in the treatment of Helicobacter pylori infection. Aliment Pharmacol Ther 1999;13:317-22.

52 Xiao SD, Liu WZ, Hu PJ, et al. High cure rate of Helicobacter pylori infection using tripotassium dicitrato bismuthate, furazolidone and clarithromycin triple therapy for 1 week. Aliment Pharmacol Ther 1999;13:311-5

53 Xiao SD, Liu WZ, Hu PJ, et al. A multicentre study on eradication of Helicobacter pylori using four 1-week triple therapies in China. Aliment Pharmacol Ther 2001;15:81-6.

54 Guo C-Y, Wu Y-B, Liu H-L, et al. Clinical evaluation of four one-week triple therapy regimens in eradicating Helicobacter pylori infection. World J Gastroenterol 2004;10:747-9.

55 Zhang Q, Hu C, Tan X, et al. Effectiveness of furazolidonecontaining triple therapies with different courses of treatment for Helicobacter pylori infection. Chinese Journal of Hospital Pharmacy 2011;31:1799-802

56 Zhang Q, Sha W, Wang Q. Therapeutic effect of clarithromycin-based triple therapy for Helicobacter pylori eradication. Chinese Journal of Gastroenterology 2012;17:480-2.

57 Chen W, Zhang G-Y, Zeng Y, et al. Furazolidone-based quadruple therapy as first-line treatment for Helicobacter pylori infection. World Chinese Journal of Digestology 2013;21:1366-71.

58 Li L, Yang W, Yang J, et al. Radical curative efficacy of quadruple therapy containing probiotics on persistent Helicobacter pylori infection: an efficacy observation. Chinese Journal of Microecology 2013;25:673-5

59 Liang X, Xu X, Zheng Q, et al. Efficacy of bismuth-containing quadruple therapies for clarithromycin-, metronidazole-, and fluoroquinolone-resistant Helicobacter pylori infections in a prospective study. Clin Gastroenterol Hepatol 2013;11:802-7.

60 Zheng X-li, Xu L. [Efficacy of furazolidone-based quadruple therapy as rescue treatment for refractory Helicobacter pylori infection]. Zhonghua Yi Xue Za Zhi 2013;93:3496-9.

$61 \mathrm{Ke}$ Q, Lu S. The efficacy of golden Bifidobacterium combined with quadruple therapy on Helicobacter pylori infection. Chinese Journal of Microecology 2018;30:695-9.

62 Fakheri H, Malekzadeh R, Merat S, et al. Clarithromycin vs. furazolidone in quadruple therapy regimens for the treatment of Helicobacter pylori in a population with a high metronidazole resistance rate. Aliment Pharmacol Ther 2001;15:411-6.

63 Yi D-M, Yang T-T, Chao S-H, et al. Comparison the cost-efficacy of furazolidone-based versus clarithromycin-based quadruple therapy in initial treatment of Helicobacter pylori infection in a variable clarithromycin drug-resistant region, a single-center, prospective, randomized, open-label study. Medicine 2019;98:e14408.

64 Daghaghzadeh H, Emami MH, Karimi S, et al. One-Week versus two-week furazolidone-based quadruple therapy as the first-line treatment for Helicobacter pylori infection in Iran. $J$ Gastroenterol Hepatol 2007;22:1399-403. 
65 Cheng H, Hu F-L. Furazolidone, amoxicillin, bismuth and rabeprazole quadruple rescue therapy for the eradication of Helicobacter pylori. WJG 2009;15:860-4.

66 Zhao R, Huo L. Efficacy of different terms and different drugs therpy for Helicobacter pylori eradication: a randmoized controlled trial. Chinese Journal of Practical Internal Medicine 2012;32:796-9.

67 Xie Y, Zhu Y, Zhou H, et al. Furazolidone-based triple and quadruple eradication therapy for Helicobacter pylori infection. World $J$ Gastroenterol 2014;20:11415-21.

68 Mokhtare M, Hosseini V, Tirgar Fakheri $\mathrm{H}$, et al. Comparison of quadruple and triple furazolidone containing regimens on eradication of Helicobacter pylori. Med J Islam Repub Iran 2015;29:195.

69 Xu X, Zhang ZY, Sun WH. Effects of pantoprazole combined with furazolidone in Helicobacter pylori eradication therapy. World Chinese Journal of Digestology 2015;23:866-71.
70 Xie Y, Zhang Z, Hong J, et al. Furazolidone-containing triple and quadruple eradication therapy for initial treatment for Helicobacter pylori infection: a multicenter randomized controlled trial in China. Helicobacter 2018;23:e12496.

71 Coelho LGV, Martins GM, Passos MCF, et al. Once-Daily, low-cost, highly effective Helicobacter pylori treatment to family members of gastric cancer patients. Aliment Pharmacol Ther 2003;17:131-6.

72 Roghani HS, Massarrat S, Shirekhoda M, et al. Effect of different doses of furazolidone with amoxicillin and omeprazole on eradication of Helicobacter pylori. J Gastroenterol Hepatol 2003;18:778-82.

73 Fakheri H, Merat S, Hosseini V, et al. Low-Dose furazolidone in triple and quadruple regimens for Helicobacter pylori eradication. Aliment Pharmacol Ther 2004:19:89-93.

74 Hooi JKY, Lai WY, Ng WK, et al. Global prevalence of Helicobacter pylori infection: systematic review and meta-analysis. Gastroenterology 2017;153:420-9. 